\title{
Evaluation of immunogenicity elicited from two DNA vaccine candidates that expresses the prM and $\mathrm{E}$ genes of the dengue- 3 virus
}

\author{
Sérgio O. de Paula ${ }^{1 * \#}$, Rafael F. O. França ${ }^{2 \star}$, Danielle M. Lima ${ }^{2}$, Nina R. Dutra ${ }^{1}$, \\ Marília B. de Paula ${ }^{1}$, Michelle D. de Oliveira ${ }^{1}$, Leandro L. de Oliveira ${ }^{1}$, \\ Benedito A. L. da Fonseca ${ }^{2}$
}

${ }^{1}$ Department of General Biology, Federal University of Viçosa, Viçosa-MG, Brazil; ${ }^{\#}$ Corresponding Author: depaula@ufv.br;
${ }^{2}$ Department of Internal Medicine, School of Medicine of Ribeirão Preto - USP, Ribeirão Preto, Brazil.

Received 18 August 2010; revised 25 August 2010; accepted 30 August 2010.

\begin{abstract}
In this work, we report the evaluation of two DNA vaccines against dengue-3 virus (DENV-3). The first construction, called pVAC3DEN3, was engineered inserting the pre-membrane (prM) and envelope (E) gene of DENV-3 truncated with a restriction site between them, as previously described. The second construction was developed cloning the full gene sequence of prM and $E$ from DENV-3 virus in $\mathrm{pCl}$ plasmid for mammalian expression and was denominated pVAC1WDEN3. The results showed that both constructions were capable of expressing the prM and $E$ proteins, as demonstrated by ELISA and immunoblotting detection in cell culture transfected with the plasmids. After positive "in vitro" results, the vaccine candidates were used to immunize BALB/c mice and the elicited response was investigated. After immunization by intramuscular inoculation with three doses of each vaccinal clone the animals were sacrificed, the cytokine levels and $T$ cell response were analyzed in the spleens, after three days of culture with stimulus, our analysis showed that the two constructions elicited $\mathrm{T}$ cell responses measured by BrdU incorporation assay and high levels of IFN-y, detected in the supernatant of the cultures. Moreover, both constructions induced detectable titers of neutralizing antibodies in mice. And finally the survival rate of the immunized animals after intracerebral challenge was analyzed, showing a better result in the pVAC3DEN3 group with an $80 \%$ survival compared with a $50 \%$ survival of the pVAC1 WDEN3.
\end{abstract}

*Both authors contributed with the same amount of work to the completion of this study.
Thus, these data showed that our two constructions were able to induce specific immune response and protects mice against a lethal challenge with DENV-3, and these vaccine candidates can be employed to develop a viable dengue vaccine.

Keywords: Dengue; DNA Vaccine; E Protein

\section{INTRODUCTION}

Immunoprophylaxis is well recognized as the most successful and widely used type of medical intervention. Some preventive vaccines, used until today, have virtually eliminated some of the worst human diseases such as polio and smallpox. However the need for vaccines against other diseases, such as dengue is urgent [1]. Dengue fever is a mosquito-borne viral disease caused by infection with the dengue virus, the disease generally has a febrile and self-limited course [2]. Infection with any of the four viruses promotes life-long immunity to the same serotype, but not to other serotypes. Thus, people living in an endemic area can acquire four different infections in their lifetime, each caused by one serotype $[3,4]$. Currently, the estimated global number of people at risk of dengue infections is approximately 2.5 billion, with almost a half million of these cases progressing to a potentially fatal syndrome known as dengue hemorrhagic fever (DHF) and dengue shock syndrome (DSS) resulting in more than 20,000 deaths per year $[5,6]$.

The expansion of dengue in the tropical areas, accompanied by a large increase of cases of DHF lead this disease to assume a public health importance, unfortunately the only available way to combat the disease is based on vector control and this measure has proven difficult and costly to sustain over time. Currently there 
are vaccines against only few human flaviviruses, the yellow fever virus, tick borne encephalitis virus and the Japanese encephalitis virus, thus the development of a vaccine against dengue is urgent and given the difficulty to achieve this, it represents a great challenge [7-9].

Given that traditional methodologies, such as viral attenuation or even viral inactivation, to develop a dengue effective vaccine were not successful, several new approaches have been planned, these include the use of molecular biology techniques mainly including improvement of DNA vaccines [10,11]. DNA vaccines offer the advantage of being secure, having a low cost production and long-term duration of immune responses [12]. Many research groups have reported the induction of immune responses in animal models, elicited by DNA vaccines, against a number of viruses including some flaviviruses [13-16]. However, it is believed that the effectiveness of DNA vaccines can be distinct from each other, even if the same antigen is targeted, since the different DNA construction strategy adopted may affect antigen presentation to the host immune system and consequently influence the elicited immune response [17,18].

The four dengue viruses are enveloped and present a spherical form with approximately a 50nm diameter; the envelope is acquired trough budding from the endoplasmic reticulum. These virions contain three structural proteins: capsid (C), membrane (M) and envelope (E) and a simple positive RNA strand genome. The membrane precursor, prM, is believed to help in the folding of the E glycoprotein and both are integrated in the lipid bilayer of the mature virion by two transmembrane regions that surround a nucleocapsid. The surface of the mature DENV is smooth with the envelope proteins aligned in pairs parallel to the virion surface. The E glycoprotein mediates cell attachment and fusion and is also the major target of protective antibodies [19,20]. In fact, the main experimental vaccines against dengue are directed to elicit immune responses against the glycoprotein E, which contains the most epitopes responsible for neutralization events [21,22].

Recently we demonstrated that the administration of a DNA vaccine, designated to express the truncated prM and $\mathrm{E}$ gene of the dengue-3 virus, was capable of inducing an immune response with the production of neutralizing antibodies and protection against intracerebral challenge in mice [23]. However, this vaccinal plasmid show- ed a mutation in the prM region. To evaluate if this mutation could compromise the immunogenicity of our con- struct, or even if the restriction site between the genes could impair the antigen presentation in vivo, in this work we constructed a new plasmid encoding the prM and E sequence of DENV-3 without restriction site between the viral glycoproteins and without mutations for test. Both constructs, the truncated and the full length sequence, were designated to express the viral glycoproteins prM and E employing the pCI plasmid for mammalian expression. Here, we compared these two constructions on their ability to induce protection in mice. Our results demonstrated that these constructions were capable of drive the expression of the viral glycoproteins in mammalian cells. In addition, these engineered vaccinal clones elicited specific antibodies in mice conferring protection against DENV-3 challenge in these animals, but surprisingly the new clone, without any mutation in all sequence, was less effective in protect mice from virus challenge and eliciting a weak immune response.

\section{METHODS}

\subsection{Cell Line, Virus, Plasmids and Animals}

C6/36, Vero and HeLa cells were purchased from the Cell Culture Section of Adolfo Lutz Institute, São Paulo, Brazil. DENV-3, H-87 strain, was kindly donated by Dr. Robert E. Shope, University of Texas at Galveston, TX. The expression plasmid (pCI) was purchased from Promega Corporation, Madison, WI. BALB/c mice, aged 2-3 week, were bred and maintained under standard conditions in the animal house of the Medical School of Ribeirão Preto, University of São Paulo, Ribeirão Preto, SP, Brazil. All animal experiments were performed in accordance with protocols approved by the School of Medicine of Ribeirão Preto Institutional Animal Care and Use Committee.

\subsection{Construction of Plasmids Expressing PrM/E Proteins}

Dengue virus RNA was purified from $0.5 \mathrm{ml}$ of a supernatant of the C6/36 cell culture infected with DENV3 using a Trizol Reagent (Invitrogen, Gaithersburg, MD) according to manufacturer's recommendations. The RNA was reverse transcribed in a standard reaction using a random hexamer primer and Superscript Mix (Invitrogen, Gaithersburg, MD). The resultant cDNA was used to amplify different segments of the virus genome, using primer pairs shown in Table 1. In order to express the DENV-3 prM/E proteins, two strategies were used. In a previous work, due to the difficulty in amplifying the whole fragment of $2044 \mathrm{pb}$, we opted to clone the viral gene by amplifying separately two fragments, the first of 1393 and a second of $651 \mathrm{pb}$, these fragments were ligated to each other by a cloning site with AccI restriction enzyme to give rise a fragment of $2044 \mathrm{pb}$ containing the prM and E viral glycoproteins, this vaccinal clone was denominated PVAC3DEN3 [23]. In thiswork, a whole fragment of $2044 \mathrm{bp}$ was amplified without any restriction 
Table 1. Construction of recombinant plasmids containing segments of dengue virus 3 genome.

\begin{tabular}{clcc}
\hline \multicolumn{1}{c}{ Primers } & \multicolumn{1}{c}{ Sequence } & Fragment & Specific Nucleotide Position \\
\hline sDEN3Mlu & 5'GGGACGCGTACATCGTGTCTCATG3' & \multirow{2}{*}{$1393 \mathrm{bp}$} & $364-1757$ \\
cDEN3Acc & 5'CCCGTCTACATTTTAAGTGCCCCG3' & & \\
sDEN3Acc & 5'GGGGTAGACTCAAGATGGACA3' & $651 \mathrm{bp}$ & $1758-2409$ \\
cDEN3Not & 5'CCCGCGGCCGCGATTCAGCTTGCACCACGACCC3' & & $364-2409$ \\
sDEN3Mlu & 5'GGGACGCGTACATCGTGTCTCATG3' & $2045 \mathrm{bp}$ & \\
cDEN3Not & 5'CCCGCGGCCGCGATTCAGCTTGCACCACGACCC3' & & \\
\hline
\end{tabular}

site between the viral glycoprotein prM and E. After cloning in the vector TOPO TA, the fragments were cloned in the pCI expression vector (Promega, Madison, WI) and the resultants plasmids were subjected to standard nucleotide sequencing.

\subsection{Nucleotide Sequencing of Plasmids Expressing PrM/E Proteins}

Sequencing primers were designed using the DENV-3, H87 strain (GenBank accession No. M93130) as the genome reference. For whole-region sequencing, PCR primer pairs were pCIs 5'CACTATAGGCTAGCCTCGAG3' and Den3AS1 5'CGCCACTGATCTATCGC3', Den3S2 5' GGCGTTAGCTCCCCATGTCG3' and Den3AS2 5'GC CATGGTAGTCACACACCC3', Den3S3 5'CCATGGC TAAGAACAAGCCC3' and Den3AS3 5'GTTTCATTT CCCACCTGGTG3', Den3S4 5'GAAACGCAGGGAG TTACGGC3' and Den3AS4 5'CCTCCTGAGGTTTGG ATCTC3', Den3S5 5'GAGATCCAAACCTCAGGAGG 3' and Den3AS5 5'CCCTTCCTGTACCAGTTGAT3', Den3S6 5'ATCAACTGGTACAGGAAGGG3' and pCIas 5'ATCATGTCTGCTCGAAGCGG3'.

The selected clones were grown at $37^{\circ} \mathrm{C}$ in an LB media with ampicilin and the plasmids were extracted using the GeneJET Plasmid Miniprep Kit (Fermentas Life Sciences, US). The plasmids were quantified by UV absorption $(260 \mathrm{~nm})$ and approximately $500 \mathrm{ng}$ of each plasmid were employed in a reaction with the ABI Prism Big Dye Terminator Cycle Sequencing Ready kit (Applied Biosystems). For each sample to be sequenced we worked with $5 \mu \mathrm{M}$ of each primer with $2 \mu \mathrm{l}$ of Big Dye, $2 \mu \mathrm{l}$ of Buffer (200 mM Tris-HCl pH 9.0 and 5mM Magnesium Chloride) and nuclease free water in a final volume of $10 \mu \mathrm{l}$. The obtained sequences were aligned using CLUSTAL W, with a final manual adjustment completed with BioEdit software and then compared with the sequences available at the Genbank.

\subsection{PrM and E Protein Expression by the Recombinant Plasmids}

PrM and E expression by the recombinant plasmids was analyzed by transfecting HeLa cells using a cationic lipid based delivery. Briefly, $30 \mu \mathrm{g}$ of plasmid DNA were mixed with Lipofectamine 2000 (Invitrogen, Gaithers- burg, MD) at a lipid mass ratio of 2:1 in $1 \mathrm{ml}$ of Minimum Essential Medium free of fetal bovine serum (FBS) and incubated for $45 \mathrm{~min}$ at room temperature. The mixture was added to cells grew to about $90 \%$ of confluence in $35 \mathrm{~mm}$ cell cultures dishes (Costar, Cambridge, MA) and incubated for $72 \mathrm{~h}$ at $37^{\circ} \mathrm{C}$ in a $5 \%$ $\mathrm{CO}_{2}$ incubator. After incubation, the cultures were processed for the detection of the prM and $\mathrm{E}$ protein expression by indirect immunofluorescence (Tesh, 1979), immunoprecipitation and a sandwich-ELISA of the culture supernants.

\subsection{Immunoprecipitation and Immuno-Blotting}

All extracts and supernatants of the transfected cells were submitted to an immunoprecipitation using a mouse immune ascitic fluid specific to DENV-3 (MIAF-DENV-3) produced in our laboratory and Sepharose Protein A (Amersham Biosciences, NJ, USA). Briefly, $1 \mathrm{ml}$ of the cellular extract and $2 \mathrm{ml}$ of the supernatant culture was added to $0.1 \mathrm{vol}$ of MIAF-DENV-3 and incubated at $4{ }^{\circ} \mathrm{C}$ for 8 hours in constant agitation. After incubation, 0.1 vol Sepharose Protein A was added to precipitate the antigen-antibody complex, and incubated at $4^{\circ} \mathrm{C}$ for 16 hours. After incubation, the complexes were recovered by centrifugation at $12.000 \mathrm{~g}$ for 30 seconds at $4^{\circ} \mathrm{C}$, washed 3 times with PBS, suspended in load buffer and submitted to SDS-PAGE. After SDS-PAGE, the proteins were transferred to a nitrocellulose membrane; the nitrocellulose membrane was blocked for 4 hours with $0.5 \%$ BSA, washed 3 times with PBS Tween-20, incubated for 2 hours at room temperature with MIAFDENV-3 (1:100), washed again, and incubated for 2 additional hours with an anti-mouse-IgG alkaline phosphatase conjugate (Sigma, Saint Louis, Missouri). The membrane was then washed 3 times with PBS Tween-20, and stained with the Western Blue Substrate for Alkaline phosphatase kit (Promega, Madison, WI).

\subsection{Densitometry Analysis of Expressed Proteins}

The densitometry analysis in detected expressed proteins was performed using the Image Processing in Analyses in JAVA-ImageJ 1.41 software (National Institute 
of Health-NIH, 2009).

\subsection{Sandwich-ELISA}

The prM and E protein expression was detected using a sandwich ELISA. Briefly, 96-well plates were coated with a high titer human antibody against dengue (1:200) and then blocked with $2 \%$ BSA. The plates were then incubated with supernatants of transfected cells that contained the expressed proteins, MIAF-DENV-3, alkaline phosphatase-conjugated anti-mouse IgG, and p-nitrophenyl phosphatase. The cut off O.D. value for determining serum positivity was calculated as the mean O.D. of the negative control sera plus 2 standard deviation (S.D.).

\subsection{Immunization of Mice with DENV-3 and Candidate Vaccines}

Groups of ten 3-week-old female Balb/c mice were injected by syringe and needle three times into the quadriceps muscle with $100 \mu \mathrm{g}$ of pVAC1WDEN3, pVAC3 DEN3 and pCI. The mice were primed on day 0 and boosted on days 10 and 30 with $100 \mu \mathrm{g}$ of DNA in a $25 \%$-PBS sucrose solution. In parallel, another group with 10 mice were immunized three times into the quadriceps muscle with $1 \times 10^{5}$ plaque-forming units per $\mathrm{ml}$ (PFU/ml) of DENV-3. Prior to boosting, blood samples were obtained through the retroorbital route. Blood samples were also obtained 10 days after the last inoculation. Sera from these mice were stored at $-70^{\circ} \mathrm{C}$ until use.

\subsection{ELISA and Plaque Reduction Neutralization Test (PRNT)}

DENV-3 antibody was detected by a solid-phase enzyme-linked immunosorbent assay (ELISA) using 96well ELISA plates coated with $100 \mu \mathrm{l}$ of DENV-1 and DENV-2 antigens (8 hemaglutination units) and incubated $\mathrm{ON}$ at $4^{\circ} \mathrm{C}$. ELISA plates were then blocked, washed and incubated with murine serum samples at 1:10 dilution in PBS for $60 \mathrm{~min}$. They were then washed three times with PBS containing 0.5\% Tween-20, and reincubated for another $60 \mathrm{~min}$ with horseradish peroxidaseconjugated goat anti-mouse IgG. Plates were washed three times and incubated with $0.1 \mathrm{M}$ sodium citrate buffer ( $\mathrm{pH}$ 5.0) containing $2.2 \mathrm{mM} \mathrm{O}$-phenylene-diamine and $0.045 \% \mathrm{H}_{2} \mathrm{O}_{2}$, and read at $490 \mathrm{~nm}$. The cutoff O.D. value for determining serum positivity was calculated as the mean O.D. of the negative control sera plus 2 S.D.

The mice serum was also assayed for DENV-3 neutralizing antibody in a plaque reduction neutralization test (PRNT) as previously described by Russell and Nisalak [24]. The percentage of plaque reduction was cal- culated for each dilution of tested sera using the number of plaques obtained with normal mouse serum as the baseline and the end-point of this assay was 1:4.096. The highest dilution of sera yielding a $50 \%$ or greater decrease in the number of plaques was considered to be the neutralization antibody titer. The statistical analysis (Tukey test with 5\% significance) was performed with GraphPad Prism 5.0 (GraphPad Software Inc, San Diego, CA).

\subsection{Quantification of Th1 Immune Response Cytokines (IFN- $\gamma$, IL-2) and Th2 Immune Response Cytokines (IL-4, IL-10) Production from Virus-Stimulated Lymphoid-Cell by ELISA}

Lymphoid cells from spleen of immunized and control mice $(\mathrm{n}=5)$ were washed twice in RPMI 1640 containing $10 \%$ heat-inactivated FBS. Cells were resuspended at a final concentration of $1 \times 10^{6}$ cells per $\mathrm{ml}$ in RPMI 1640 and $100 \mu \mathrm{l}$ aliquots were plated into 96-well culture plates. Then, $1 \times 10^{5} \mathrm{PFU} / \mathrm{ml}$ of heat inactivated DENV-3 was added to each well to a final volume of $200 \mu \mathrm{l}$; plates were covered and incubated at $37^{\circ} \mathrm{C}$ in a $5 \% \mathrm{CO}_{2}$ atmosphere. Following stimulation, aliquots of supernatants were removed after $48 \mathrm{~h}$ and stored at $-70^{\circ} \mathrm{C}$ for subsequent analysis. Sandwich-type ELISA (DuoSet $^{\mathrm{TM}}, \mathrm{R} \& \mathrm{D}$ Systems, MN) were used to estimate the IFN- $\gamma$, IL-2, IL-4 and IL-10 levels in the supernatants of virus-stimulated cells, according to manufacturer's instructions. Briefly, serial dilutions of cytokine standards, samples and controls were added into 96-well microplates coated with specific monoclonal antibody and incubated for $2 \mathrm{~h}$ at room temperature. Plates were then washed five times with $\mathrm{PBS} / \mathrm{T}$ (PBS/0.5\% Tween) and $100 \mu \mathrm{l}$ of horseradish-peroxidase-linked polyclonal antibody specific for mouse cytokines were added. After $2 \mathrm{~h}$ of incubation at room temperature, the plates were washed five times and $100 \mu$ of a substrate solution were added per well. After $30 \mathrm{~min}$ incubation at room temperature, the plates were read at $450 \mathrm{~nm}$. Levels of cytokines in the supernatants were calculated based on the comparison of their OD with the standard calibration curve. The statistical analysis (Tukey test with 5\% significance) was performed with GraphPad Prism 5.0 (GraphPad Software Inc, San Diego, CA).

\subsection{T Cell Proliferation Assay}

The DENV-3 specific lymphoproliferative responses from DNA-immunized mice were determined by Cell Proliferation ELISA (BrdU Lymphoproliferation kit Ro- 
che, Mannheim, Germany). Spleens were prepared from recombinant pVAC1WDEN3 pVAC3DEN3, DENV-3, and pCI-inoculated from 5 mice per group. Cell suspensions were treated with Tris-buffered ammonium chloride to eliminate the red blood cells, washed, and resuspended in RPMI 1640 supplemented with 5\% FBS, HEPES buffer, L-glutamine, penicillin and streptomycin. Cells were cultured in triplicate in 96-well microtiter plates $\left(1 \times 10^{6}\right.$ cells $/ 200 \mu$ l per well $)$ in the presence of heat inactivated DENV-3 $\left(1 \times 10^{4} \mathrm{PFU} / \mathrm{ml}\right.$ or $1 \times 10^{6}$ $\mathrm{PFU} / \mathrm{ml})$, control RPMI medium, and ConA $(0.1 \mu \mathrm{g} / \mathrm{ml})$. After $72 \mathrm{~h}$, cultures were pulsed with $10 \mu \mathrm{M}$ BrdU and incubated for $24 \mathrm{~h}$ at $37^{\circ} \mathrm{C}$. The labeling medium was then removed by suction and the plate was dried at $60^{\circ} \mathrm{C}$ for $1 \mathrm{~h}$. The cells were fixed with FixDenat solution, incubated with anti-BrdU POD antibody, and the antigen-antibody reaction was detected by the subsequent substrate reaction read at $450 \mathrm{~nm}$. The statistical analysis, Two-way Anova followed by Bonferroni post test, was performed with GraphPad Prism 5.0 (GraphPad Software Inc, San Diego, CA).

\subsection{Challenge Experiments in Mice}

Groups of 10 3-week-old female Balb/c mice, were immunized with $100 \mu \mathrm{g}$ of recombinant pVAC1WDEN3, pVAC3DEN3 and pCI DNA in sucrose 25\%-PBS. Recombinant clones were intramuscularly injected into the quadriceps of the mice and boosted with $100 \mu \mathrm{g}$ DNA 10 and 20 days later. A group with 10 mice was also immunized with $1 \times 10^{4} \mathrm{PFU} / \mathrm{ml}$ of DENV-3 intraperitonially and boosted on the same scheduled dates. Twenty-one days after the third inoculation, mice were challenged intracerebrally with $50 \mathrm{LD}_{50}\left(1 \times 10^{5} \mathrm{PFU} / \mathrm{ml}\right)$ of DENV-3, prepared from DENV-3-infected suckling mice brains, and mouse survival was monitored daily for 21 days. The statistical analysis (Long-Rank test, Mantel-Cox) was performed with GraphPad Prism 5.0 (GraphPad Software Inc, San Diego, CA).

\section{RESULTS}

\subsection{Nucleotide Sequencing of Plasmids Expressing PrM/E Proteins}

When the pVAC3DEN3 clone amino acid sequence was compared with the published reference sequence, we found a single mutation in the region of the prM gene, replacing $\mathrm{F}$ to $\mathrm{C}$ in position 39 of the amino acid sequence. With the objective of improving our DNA vaccine, we chose a clone that didn't demonstrate any mutation in the $\mathrm{prM} / \mathrm{E}$ genes. This clone originated from the whole fragment amplification and was called pVAC1
WDEN3.

\subsection{Expression of PrM and E DENV-3 Recombinant Proteins}

Two recombinant plasmids, pVAC1WDEN3 e pVAC3 DEN3, were selected to be evaluated. They all contained an ATG codon and a translation initiation site provided by the forward primers used in the PCR amplification. They were all designed to express prM and $\mathrm{E}$ proteins. Cells transfected with all two DNA constructs showed positive IFA with MIAF-DENV-3, while cells transfected with pCI were negative (data not shown). As showed on Figure 1, a band with a molecular weight of 53-54 kDa, which correlated with the expected E protein molecular weight, was detected in cell lysates by immunoprecipitation followed by immunoblotting. Densitometry analysis of the immunoblotting showed no significance difference between pVAC3DEN3 and pVAC1 WDEN3 in the prM/E protein expression on intracellular fractions (data not shown). Examination of supernatants by sandwich-ELISA from transfected cells revealed that the expressed proteins from all 2 clones were also secreted into the supernatant (Figure 1).

\subsection{Antibody Response in Immunized Mice}

Ten mice per group were inoculated with $100 \mu \mathrm{g}$ of each of the three DNA constructs, DENV-3 and pCI, as described in the methods. In all groups neutralizing antibody titers were detected, induced by candidate DNA vaccines in comparison to the antibody titers observed in the DENV-3 inoculated mice and no statistical difference was detected among the groups (Figure 2).

\subsection{Cytokine Response in Immunized Mice}

ELISA results showed that IFN- $\gamma$ and IL-2 were synthesized by the lymphocyte cells of mice immunized with pVAC3DEN3 and IFN- $\gamma$, IL-2 and IL-10 were synthesized by the lymphocyte cells of immunized mice with pVAC1WDEN3 as shown in Table 2. Spleen cells of DENV-3 immunized mice produced all four cytokines tested, demonstrating the ability of a natural infection in

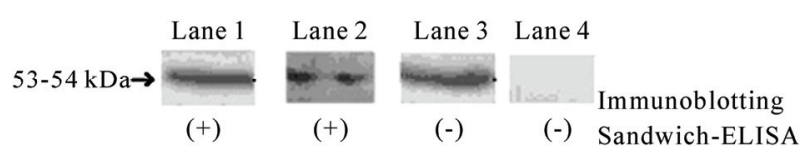

Figure 1. Analysis of DENV-3 prM/E protein expression on intracellular fractions by immunoprecipitation followed by Immunoblotting. HeLa cells were transfected with $30 \mu \mathrm{g}$ of purified pVAC3DEN3 (Lane 1) or pVAC1WDEN3 (Lane 2), DENV-3 M.O.I = 1 (Lane 3) and pCI as negative control (Lane 4). 
Table 2. Quantification of Th1 immune response cytokines (IFN- $\gamma$ ) and Th2 immune response cytokines (IL-4) of mice recipients of DNA vaccines.

\begin{tabular}{|c|c|c|c|c|c|}
\hline & & IFN- $\gamma$ & IL-2 & IL-4 & IL-10 \\
\hline & stimuli & $\mathrm{pg} / \mathrm{ml}$ & $p g / m l$ & $\mathrm{pg} / \mathrm{ml}$ & $p g / m l$ \\
\hline pVAC1WDEN3 & $D E N V-3$ & $361.54 \pm 0.2$ & $11.75 \pm 1.2$ & 0.0 & $93.4 \pm 2.1$ \\
\hline pCI & $D E N V-3$ & 0.0 & 0.0 & 0.0 & 0.0 \\
\hline DENV-3 & $D E N V-3$ & $75.45 \pm 1.6$ & $48.25 \pm 0.5$ & $28.13 \pm 1.7$ & $16.89 \pm 1.4$ \\
\hline pVAC3DEN3 & $D E N V-3$ & $77.5 \pm 1.5$ & $445.73 \pm 3.2$ & 0.0 & 0.0 \\
\hline pCI & $D E N V-3$ & 0.0 & 0.0 & 0.0 & 0.0 \\
\hline DENV-3 & $D E N V-3$ & $125.42 \pm 1.6$ & $123.75 \pm 1.35$ & $10.32 \pm 1.35$ & $56.89 \pm 0.8$ \\
\hline
\end{tabular}

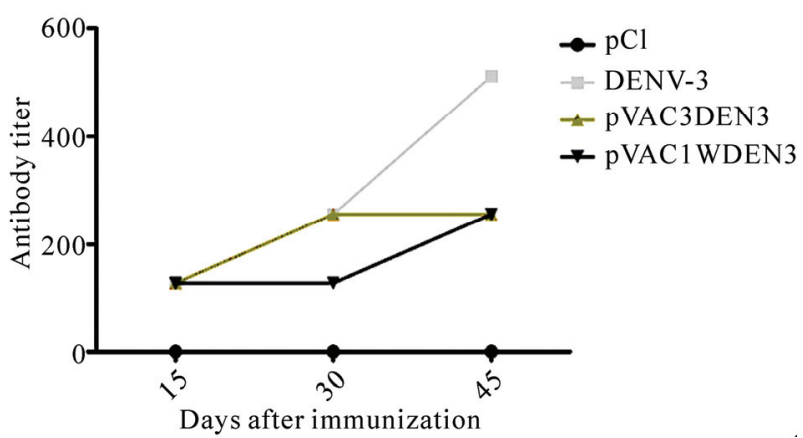

Figure 2. Plaque reduction neutralization test.

inducing a potent immune response. The high levels of IFN- $\gamma$ in the vaccinated groups indicate a Th1 pattern response.

\subsection{DENV-3-Specific T Cell Proliferation in DNA Vaccinated Mice}

We evaluated if the plasmid DNA immunization could induce DENV-3-specific lymphoproliferative response in Balb/c mice splenocytes, cultivated and assayed with $\mathrm{BrDu}$ in response to specific antigen stimulation. Splenic lymphocytes derived from pVAC1WDEN3 and pVAC3 DEN3-inoculated animals demonstrated a dose-dependent proliferative response to inactivated DENV-3, as shown in Figure 3. Proliferation responses were always higher than the negative control, and the response of both vaccine candidates to a higher dose of antigen was comparable to that observed in DENV-3 immunized mice.

In addition the response of pVAC3DEN3 immunized group was higher than pVAC1WDEN3 $(p<0.05)$ in the cells stimulated with $10^{6} \mathrm{PFU}$ of dengue virus, suggesting a better immunogenicity of this construct.

\subsection{Challenge of Immunized Mice}

PVAC1WDEN3 and pVAC3DEN3 vaccine candidates were evaluated in accordance to their ability to induce protective immunity against lethal challenge with DENV3. Groups of 10, three week-old Balb/c mice, were immunized with the DNA vaccines, and positive and negative control mice were immunized with $1 \times 10^{4} \mathrm{PFU} / \mathrm{ml}$ of DENV-3 and with $100 \mu \mathrm{g}$ of pCI, respectively. As shown in Figure 4, immunization with the pVAC3DEN3 induced a solid protection against the DENV-3 challenge comparable to that observed in DENV-3 inoculated mice, where $80 \%$ of the challenged mice survived. However, only $50 \%$ survival was observed after immunization with pVAC1WDEN3 ( $p=0.304$ compared to $p C I)$. The negative control group, immunized with $\mathrm{pCI}$, presented approximately $20 \%$ survival, as expected for negative control group. Only the pVAC3DEN3 immunized group showed statistical signifance in challenge protection when compared with pCI.

\section{DISCUSSION}

In general, the DNA vaccines presents many advantages over other immunization conventional strategies, these include: easiness of production, stability and transport at room temperature, decreased likelihood of replication interference and the possibility to vaccinate against multiple pathogens in a single vaccination [1]. In an effort to develop a DNA vaccine for dengue virus, based on the envelope viral glycoproteins prM and E, we expanded our previous work with the pCI plasmid. In a previous work we constructed a DNA vaccine candidate inserting the prM and subsequently the $\mathrm{E}$ gene of dengue virus type 3 , separated by a restriction site, so the protein was expressed with the restriction site between the junction of prM and E. We showed that this construction named pVAC3DEN3 was capable of inducing protection in $80 \%$ of the immunized animals, after challenge [23]. In the present report, we compared the response elicited by two distinct construction methodologies. Now a second 


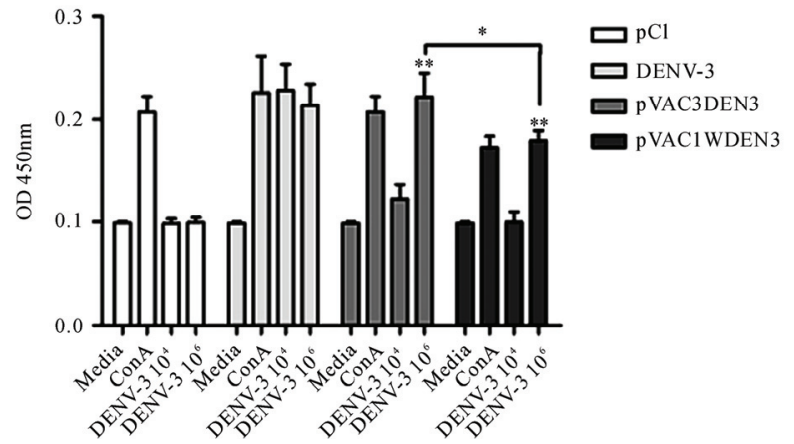

Figure 3. Proliferation responses to dengue virus in mice receiving the DNA vaccines. Media, negative control (RPMI Medium as stimulus); DENV3-10 $0^{4} 1 \times 10^{4} \mathrm{PFU} / \mathrm{ml}$ of heat inactivated virus; DENV-3 106 : $1 \times 10^{6} \mathrm{PFU} / \mathrm{ml}$ of heat inactivated virus; ConA: Concanavalin A. ${ }^{*} \mathrm{p}<0.001$ when compared to pCI control group, *p $<0.05$ when compared the pVAC3DEN3 versus pVAC1WDEN3 immunized group (Twoway ANOVA, Bonferroni post test).

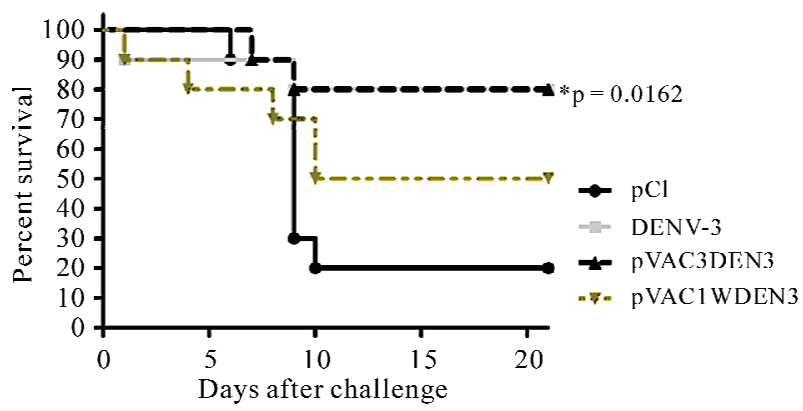

Figure 4. Survival of DNA-immunized mice after challenge with a lethal dose of DENV-3. There was no statistic difference between pVAC3DEN3 and pVAC1WDEN3 challenged groups $p=0.182$ and on $p C I$ versus $\mathrm{pVAC} 1 \mathrm{WDEN} 3 \mathrm{p}=0.304$, on $\mathrm{pCI}$ versus $\mathrm{pVAC} 3 \mathrm{DEN} 3\left({ }^{*} \mathrm{p}=0.0162\right)$ the protection was significant (Log-rank Test, Mantel-Cox).

plasmid denominated pVAC1WDEN3 was engineered, containing again the $\mathrm{prM} / \mathrm{E}$ genes, however the full sequence of the genes were inserted in the plasmid without any cloning site between them and their immunogenicity was evaluated and compared.

Expression of the recombinant protein in DNA transfected mammalian cells was analyzed after three days of transfection. As revealed by immunobloting and ELISA, the two constructions were able to express the protein, either secreted on the supernatant culture or cell associated. Apparently the constructions were functional when analyzed in vitro, directioning the protein expression that can be recognized by specific antibodies. The fact that there were no difference in expression between the constructs, when analized by densitometry of the bands in immunobloting, suggests that the mutation in the prM region of the pVAC3DEN3 clone did not impaired the protein expression.
Our data of protein expression by transfected cells is in agreement with previous works that demonstrate the prM gene is necessary for the correct expression of the $\mathrm{E}$ protein genes [9,25-27]. The prM protein consists of approximately 165 amino acids and is accepted that it might function as a chaperone for folding and assembly of the E protein [28]. In a work published by Jimenez and Fonseca [29], in the groups of mice inoculated intramuscularly with a recombinant plasmid expressing only the E protein of dengue virus 2, containing $94 \%$ of the $\mathrm{E}$ gene, no response with anti-dengue antibodies, cellular proliferation, or synthesis of cytokines by their lymphoid cells were observed. However, protection was observed in $20 \%$ of the challenged mice immunized with this recombinant plasmid and the mice survived longer than the control group. The authors speculate that the low percentage of protection might be explained by a weak activation of the immune system resulting from an imperfect secretion of $\mathrm{E}$ protein due to the lack of the prM protein. Our concern about the mutation in the prM region of pVAC3DEN3, was if this mutation could impair the expression level of the $\mathrm{E}$ protein, resulting in week immune stimulation, to address if this could be possible we constructed a new plasmid encoding these viral genes and selected a construct without mutations.

In our results, the levels of $\mathrm{T}$ cell proliferation after stimulus and even cytokines secretion were similar between the two constructions, either pVAC3DEN3 or pVAC1WDEN3. Although, the construction pVAC3 DEN3 showed a mutation in the prM region, position 39 replacing $\mathrm{F}$ to $\mathrm{C}$ in the amino acid sequence, this construct showed a better lymphoproliferative response in response to dengue stimulus when compared to pVAC1 WDEN3. Briefly, we showed the efficacy of both our plasmid constructions in express the viral glycoproteins, assessed in vitro.

There is a consensus that the induction of neutralizing antibodies directed against the virus envelope (E) protein is the most important mediator of protection against dengue infection, thus the induction of protective levels of neutralizing antibodies is the key of successful immunization [1]. Here, we tested the protection efficacy of the candidate vaccines by detecting the levels of neutralizing antibodies, the animals vaccinated with pVAC3 DEN3 and pVAC1WDEN3 showed an end point neutralizing titer of 256 . The titers of the vaccinated animals were near to the titers observed in the animals that were inoculated with the DENV-3, these neutralizing antibodies indicated the induction of humoral immune responses in the experimental immunization. In a study with recombinant MVA (modified vaccinia, Ankara) expressing only a dengue- 2 truncated E protein, $\mathrm{PRNT}_{50}$ titers of 70 and higher as being protective was reported [30]. How- 
ever, other authors reported that low antibody titers between 20 and 80 were also found protective against dengue challenge [31]. Wherefore, we believe that our neutralizing antibodies titer, in mice, is sufficient to induce a good protection against infection.

We analyzed $\mathrm{T}$ cell responses by the BrdU incorporation assay using heat inactivated dengue- 3 virus as stimulus. In this work, we showed that all the recombinant plasmids were immunogenic and elicit $\mathrm{T}$ cell proliferation after 3 days of stimulation in vitro. Although most of the studies in dengue vaccine development are focused in analyzing only the generation of neutralizing antibodies, some authors are interested in study $\mathrm{T}$ cell responses. Khanam et al. 2006 [32] showed that splenocytes, obtained from immunized mice in response to antigen stimulation in vitro, manifested a significant proliferative response accompanied by the production of high levels of IFN- $\gamma$, after immunization with a vaccine candidate to dengue virus type 2 envelope domain III encoded by plasmid and adenoviral vectors in a prime/ boost strategy. Probably the major focus in antibodies reflects the scantiness in data about $\mathrm{T}$ cells responses in dengue infections. In our vaccinal strategy, the response to stimulus in both vaccine candidates ( $p$ VAC1WDEN3 and pVAC3DEN3) cultivated spleen cells, produced a high quantity of IFN- $\gamma$ in comparison of IL-4 production. IL-4 is a B-cell stimulatory cytokine and contributes markedly to the generation of a dengue virus-neutralizing antibody response [32]. However the critical question that we addressed and showed is that our neutralizing antibody titers were protective in both vaccines, even without IL-4 detection. These results are in agreement with another study where the levels of IFN- $\gamma$ were higher than IL-4, obtained from splenocytes in proliferative response after immunization with a plasmid enconding the domain III of the dengue 2 E protein [32].

Dengue virus is not usually pathogenic to mice. Although some studies have demonstrated that commonly used laboratory mouse strains are permissive to dengue virus infection and even replication, no overt signs of disease are observed in these animals and wild type viruses replicate to such low titers in mouse tissues that they are scarcely detectable [33]. Therefore, the lack of an appropriated animal model to test vaccine candidates is one of the major obstacles in the field of dengue research and vaccine development. The mouse model predominately used to test the efficacy of DENV vaccines is based in intracerebral infection of mice with mousebrain-adapted DENV [34-37]. Employing the model of intracerebral challenge our animals vaccinated with pVAC3DEN3 obtained a high survival rate after challenge $(80 \%)$. The control group inoculated with the DE-
NV-3 also showed an $80 \%$ protection, while the group inoculated with the pCI plasmid obtained a $20 \%$ protection, the group that received immunization with pVAC1WDEN3 showed a survival of 50\%. This difference between the vaccinated groups was not surprising, once that the model of intracerebral challenge does not represent a natural infection some variation can be expected. It's largely discussed by several authors the lack of a good animal model to test dengue vaccines and some groups have focused it is efforts to achieve a better laboratory model [38]. Here we believe that our vaccine evaluation could be impaired by the mouse model employed explaining the low level of protection in the pVAC1WDEN3 immunized group.

In an attempt to improve our vaccine, we constructed another plasmid expressing the same genes, evaluating and comparing the immunogenicity of both constructions. In Brazil, the dengue virus represents a health public problem of great importance since that each summer dengue outbreak occurs in several urban centers. Thus, considering that the immune response induced by pVAC3DEN3, in comparison with pVAC1WDEN3 vaccine candidate, and considering the work being carried out with the other dengue viruses by our group, this vaccine candidate will certainly be analyzed in a tetravalent DNA vaccine format to determine the vaccine efficacy.

\section{ACKNOWLEDGEMENTS}

This work was supported by Fundação de Amparo a Pesquisa do Estado de São Paulo (FAPESP), São Paulo, Brazil. SOP was supported by a FAPESP scholarship.

\section{REFERENCES}

[1] Whitehead, S.S., Blaney, J.E., Durbin, A.P. and Murphy, B.R. (2007) Prospects for a dengue virus vaccine. Nature Reviews Microbiology, 5, 518-528.

[2] Halstead, S.B. (1989) Antibody, macrophages, dengue virus-infection, shock, and hemorrhage: A pathogenetic cascade. Reviews of Infectious Diseases, 11, S830-S839.

[3] Innis, B.L. (1995) Dengue and dengue hemorrhagic fever. In: Porterfield, J.S. Ed., Exotic Viral Infections, Chapman Hall, London, 103-146.

[4] Kuno, G. (2004) Serodiagnosis of flaviviral infections and vaccinations in humans. Advances in Virus Research, 61, 63-65.

[5] Gubler, D.J. (1998) Population growth, urbanization, automobiles and aeroplanes: The dengue connection. In: Greenwood, B. and De Cock, K. Eds., New \& Resurgent Infection. Prediction, Detection \& Management of Tomorrows Epidemics, John Wiley \& Sons, New York, 117129.

[6] Gubler, D.J. (2002) The global emergence/resurgence of arboviral diseases as public health problems. Archives of Medical Research, 33, 330-342. 
[7] Barrett, A.D.T. (1997) Yellow fever vaccines. Biologicals, 25, 17-25.

[8] Barrett, A.D.T. (1997) Japanese encephalitis and dengue vaccines. Biologicals, 25, 27-34.

[9] Raviprakash, K., Kochel, T.J., Ewing, D., Simmons, M., Phillips, I., et al. (2000) Immunogenicity of dengue virus type 1 DNA vaccines expressing truncated and full length envelope protein. Vaccine, 18, 2426-2434.

[10] Edelman, R. (2005) Dengue and dengue vaccines. Journal of Infectious Diseases, 191, 650-653.

[11] Kinney, R.M. and Huang, C.Y.H. (2001) Development of new vaccines against dengue fever and Japanese encephalitis. Intervirology, 44, 176-197.

[12] Imoto, J., Konishi, E. (2007) Dengue tetravalent DNA vaccine increases its immunogenicity in mice when mixed with a dengue type 2 subunit vaccine or an inactivated Japanese encephalitis vaccine. Vaccine, 25, 10761084.

[13] Colombage, G., Hall, R., Pavy, M. and Lobigs, M. (1998) DNA-based and alphavirus-vectored immunisation with prM and E proteins elicits long-lived and protective immunity against the flavivirus, Murray Valley encephalitis virus. Virology, 250, 151-163.

[14] Konishi, E., Yamaoka, M., Khin Sane, W., Kurane, I. and Mason, P.W. (1998) Induction of protective immunity against Japanese encephalitis in mice by immunization with a plasmid encoding Japanese encephalitis virus premembrane and envelope genes. Journal of Virology, 72, 4925-4930.

[15] Phillpotts, R.J., Venugopal, K. and Brooks, T. (1996) Immunisation with DNA polynucleotides protects mice against lethal challenge with St Louis encephalitis virus. Archives of Virology, 141, 743-749.

[16] Schmaljohn, C., Vanderzanden, L., Bray, M., Custer, D., Meyer, B., et al. (1997) Naked DNA vaccines expressing the prM and $\mathrm{E}$ genes of Russian spring summer encephalitis virus and Central European encephalitis virus protect mice from homologous and heterologous challenge. Journal of Virology, 71, 9563-9569.

[17] Alves, A.M.B., Lasaro, M.O., Almeida, D.F. and Ferreira, L.C.S. (1999) New vaccine strategies against enterotoxigenic Escherichia coli. I: DNA vaccines against the CFA/I fimbrial adhesin. Brazilian Journal of Medical and Biological Research, 32, 223-229.

[18] Donnelly, J.J., Wahren, B. and Liu, M.A. (2005) DNA vaccines: Progress and challenges. Journal of Immunology, 175, 633-639.

[19] Mukhopadhyay, S., Kuhn, R.J., Rossmann, M.G. (2005) A structural perspective of the Flavivirus life cycle. $\mathrm{Na}$ ture Reviews Microbiology, 3, 13-22.

[20] Kuhn, R.J., Zhang, W., Rossmann, M.G., Pletnev, S.V., Corver, J., et al. (2002) Structure of dengue virus: Implications for flavivirus organization, maturation, and fusion. Cell, 108, 717-725.

[21] Brinton, M.A., Kurane, I., Mathew, A., Zeng, L.L., Shi, P.Y., et al. (1998) Immune mediated and inherited defences against flaviviruses. Clinical and Diagnostic Virology, 10, 129-139.

[22] Chambers, T.J., Hahn, C.S., Galler, R. and Rice, C.M. (1990) Flavivirus genome organization, expression, and replication. Annual Review of Microbiology, 44, 649-688.

[23] De Paula, S.O., Lima, D.M., Oliveira França, R.F., Go-
mes-Ruiz, A.C. and Fonseca, B.A.L (2008) A DNA vaccine candidate expressing dengue- 3 virus prM and E proteins elicits neutralizing antibodies and protects mice against lethal challenge. Archives of Virology. 153(12), 2215-2223.

[24] Russell, P.K. and Nisalak, A. (1967) Dengue virus identification by plaque reduction neutralization test. Journal of Immunology, 99, 291.

[25] Konishi, E., Pincus, S., Paoletti, E., Shope, R.E., Burrage, T., et al. (1992) Mice immunized with a subviral particle containing the Japanese Encephalitis-virus prM/M and E-proteins are protected from lethal JEV infection. $V i$ rology, 188, 714-720.

[26] Fonseca, B.A.L., Pincus, S., Shope, R.E., Paoletti, E. and Mason, P.W. (1994) Recombinant Vaccinia viruses coexpressing Dengue-1 glycoproteins prM and E-induce neutralizing antibodies in mice. Vaccine, 12, 279-285.

[27] Allison, S.L., Stadler, K., Mandl, C.W., Kunz, C., Heinz, F.X. (1995) Synthesis and secretion of recombinant TickBorne Encephalitis-Virus protein-E in soluble and particulate form. Journal of Virology, 69, 5816-5820.

[28] Lorenz, I.C., Allison, S.L., Heinz, F.X., Helenius, A. (2002) Folding and dimerization of tick-borne encephalitis virus envelope proteins prM and $\mathrm{E}$ in the endoplasmic reticulum. Journal of Virology, 76, 5480-5491.

[29] Jimenez, R.O. and da Fonseca, B.A.L. (2000) Recombinant plasmid expressing a truncated dengue- 2 virus $\mathrm{E}$ protein without co-expression of prM protein induces partial protection in mice. Vaccine, 19, 648-654.

[30] Men, R., Wyatt, L., Tokimatsu, I., Arakaki, S., Shameem, G., et al. (2000) Immunization of rhesus monkeys with a recombinant of modified vaccinia virus Ankara expressing a truncated envelope glycoprotein of dengue type 2 virus induced resistance to dengue type 2 virus challenge. Vaccine, 18, 3113-3122.

[31] Guirakhoo, F., Pugachev, K., Zhang, Z., Myers, G. and Levenbook, I., et al. (2004) Safety and efficacy of chimeric yellow fever-dengue virus tetravalent vaccine formulations in nonhuman primates. Journal of Virology, 78, 4761-4775.

[32] Khanam, S., Khanna, N. and Swarninathan, S. (2006) Induction of neutralizing antibodies and $\mathrm{T}$ cell responses by dengue virus type 2 envelope domain III encoded by plasmid and adenoviral vectors. Vaccine, 24, 6513-6525.

[33] Bente, D.A. and Rico-Hesse, R. (2006) Models of dengue virus infection. Drug Discovery Today: Disease Models, 3(1), 97-103.

[34] Kaufman, B.M., Summers, P.L., Dubois, D.R. and Eckels, K.H. (1987) Monoclonal-antibodies against Dengue-2 virus E-glycoprotein protect mice against lethal dengue infection. American Journal of Tropical Medicine and Hygiene, 36, 427-434.

[35] Bray, M., Zhao, B.T., Markoff, L., Eckels, K.H., Chanock, R.M., et al. (1989) Mice immunized with recombinant vaccinia virus expressing Dengue-4 virus structural proteins with or without nonstructural protein-NS1 are protected against fatal Dengue virus encephalitis. Journal of Virology, 63, 2853-2856.

[36] Falgout, B., Bray, M., Schlesinger, J.J. and Lai, C.J. (1990) Immunization of mice with recombinant Vaccinia virus expressing authentic Dengue virus nonstructural protein NS1 protects against lethal Dengue virus en- 
cephalitis. Journal of Virology, 64, 4356-4363.

[37] Van Der Most, R.G. and Strauss, J.H. (2000) Chimeric yellow fever/dengue virus as a candidate dengue vaccine: Quantitation of the dengue virus-specific CD8 T-cell re- sponse. Journal of Virology, 74, 8094-8101.

[38] Yauch, L.E. and Shresta, S. (2008) Mouse models of dengue virus infection and disease. Antiviral Research, 80, 87-93. 